\title{
PERFIL SOCIODEMOGRÁFICO E CLÍNICO DOS PACIENTES EM TRATAMENTO NA UNIDADE PSIQUIÁTRICA DE UM HOSPITAL GERAL*
}

Thaise Liara da Silva', Mariluci Alves Maftum², Luciana Puchalski Kalinke², Thais Aidar de Freitas Mathias ${ }^{4}$, Aline Cristina Zerwes Ferreira ${ }^{5}$, Fernanda Carolina Capistrano ${ }^{6}$

${ }^{1}$ Enfermeira. Mestre em Enfermagem. Enfermeira do Instituto Federal Goiano. Morrinhos-GO-Brasil.

${ }^{2}$ Enfermeira. Doutora em Enfermagem. Docente da Universidade Federal do Paraná. Curitiba-PR-Brasil.

${ }^{3}$ Enfermeira. Doutora em Ciências da Saúde. Docente da Universidade Federal do Paraná. Curitiba-PR-Brasil.

${ }^{4}$ Enfermeira. Doutora em Saúde Pública. Docente da Universidade Estadual de Maringá. Maringá-PR-Brasil.

${ }^{5}$ Enfermeira. Mestranda em Enfermagem. Universidade Federal do Paraná. Curitiba-PR-Brasil.

${ }^{6}$ Enfermeira. Doutoranda em Enfermagem. Universidade Federal do Paraná. Curitiba-PR-Brasil.

RESUMO: Pesquisa descritiva transversal realizada em uma Unidade Psiquiátrica de um hospital geral de Guarapuava, Paraná, com o objetivo de caracterizar o perfil sociodemográfico e clínico dos pacientes com transtorno mental, em tratamento em unidade de internação psiquiátrica de um hospital geral. Participaram da pesquisa 240 pacientes internados no primeiro semestre de 2011. Os dados foram analisados por frequências e percentuais e mostrados em tabelas. Os resultados apontam para um perfil de pessoas brancas, solteiras, em idade produtiva, com baixa escolaridade, com pelo menos um filho. Evidenciou-se prevalência do desenvolvimento dos transtornos mentais por mais de 20 anos, com início na adolescência, com destaque para o diagnóstico de transtornos mentais e comportamentais devidos ao uso substâncias psicoativas. Conclui-se que conhecer o perfil sociodemográfico e clínico dos pacientes possibilita o desenvolvimento de ações de saúde e o fortalecimento de relações multiprofissionais e intersetoriais.

DESCRITORES: Enfermagem; Saúde mental; Perfil de saúde; Serviços de saúde mental.

\section{SOCIODEMOGRAPHIC AND CLINICAL PROFILE OF PATIENTS TREATED AT THE PSYCHIATRIC UNIT OF A GENERAL HOSPITAL}

\begin{abstract}
Descriptive and cross-sectional research undertaken at a Psychiatric Unit of a general hospital in Guarapuava, Paraná, to characterize the sociodemographic and clinical profile of patients with mental disorders being treated at a psychiatric ward of a general hospital. The research participants were 240 patients hospitalized in the first semester of 2011. The data were analyzed using frequencies and percentages and displayed in tables. The results indicate a profile of white, single people of productive age, with low education levels and at least one child. The prevalent period for the development of the mental disorders was more than 20 years earlier, starting in adolescence. The diagnosis of mental and behavioral disorders due to psychoactive substance use stood out. In conclusion, knowing the patients' sociodemographic and clinical profile permits the development of health actions and the strengthening of multiprofessional and intersectoral relationships.
\end{abstract}

DESCRIPTORS: Nursing; Mental health; Health profile; Mental health services.

\section{PERFIL SOCIODEMOGRÁFICOY CLÍNICODELOS PACIENTES EN TRATAMIENTO EN LA UNIDAD PSIQUIÁTRICA DE UN HOSPITAL GENERAL}

RESUMEN: Investigación descriptiva transversal realizada en una Unidad Psiquiátrica de un hospital general de Guarapuava, Paraná, con la finalidad de caracterizar el perfil sociodemográfico y clínico de los pacientes con trastorno mental, en tratamiento en unidad de internación psiquiátrica de un hospital general. Participaron de la investigación 240 pacientes internados en el primer semestre de 2011. Los datos fueron analizados por frecuencias y percentuales y organizados en tablas. Los resultados apuntan para un perfil de personas blancas, solteras, en edad productiva, con baja escolaridad, con por lo menos un hijo. Se evidenció prevalencia del desarrollo de los trastornos mentales por más de 20 años, con inicio en la adolescencia, destacándose el diagnóstico de trastornos mentales y comportamentales advenidos del uso de substancias psicoativas. Se concluye que conocer el perfil sociodemográfico y clínico de los pacientes posibilita el desarrollo de acciones de salud y el fortalecimiento de relaciones multiprofesionales e intersectoriales.

DESCRIPTORES: Enfermería; Salud mental; Perfil de salud; Servicios de salud mental.

*Artigo extraído de Dissertação intitulada: Perfil sociodemográfico, clínico e de internação de pacientes em tratamento na unidade psiquiátrica de um hospital geral, Universidade Federal do Paraná. Curitiba, 2011. 


\section{INTRODUÇÃO}

Os transtornos mentais estão entre as condições crônicas mais prevalentes, podendo afetar indivíduos de qualquer classe social e em qualquer fase da vida. Dados epidemiológicos da Organização Mundial de Saúde mostram que, aproximadamente, 650 milhões de pessoas no mundo sofrem de algum tipo de transtorno mental, ocasionando grande sofrimento individual, familiar e social(1).

Estima-se que mais de $10 \%$ da carga global de todas as doenças do mundo, mensurada em termos de tempo perdido de vida saudável, pode ser atribuída a transtornos mentais. Ao considerar o fator incapacidade, estes transtornos são responsáveis, em todo o mundo, por $25 \%$ da soma dos anos vividos com incapacidade ${ }^{(1)}$. Esses dados caracterizam o transtorno mental como importante contribuinte para a morbidade e a mortalidade prematura.

No Brasil, o Ministério da Saúde informa que cerca de $3 \%$ da população geral sofre de transtornos mentais severos e persistentes, 6\% da população apresenta transtornos psiquiátricos pelo uso de álcool e drogas e $12 \%$ requer atendimentos em saúde mental contínuo ou eventual( ${ }^{(2)}$. Para atender a crescente demanda foi instituída a Rede de Atenção Psicossocial no Sistema Único de Saúde (RAPS), que prioriza os serviços de base comunitária para reinserção do indivíduo na sociedade e o resgate da sua cidadania. Para tanto, preconizam-se a articulação e a integração entre os diferentes pontos de atenção como Unidades Básicas de Saúde, Centros de Atenção Psicossocial, Serviços de Urgência e Emergência e Unidades Psiquiátricas em Hospitais Gerais (UPHG) ${ }^{(3)}$.

Quando os recursos disponíveis nos serviços comunitários são insuficientes, para assistir de modo efetivo a pessoa com transtorno mental, torna-se necessária a internação hospitalar. Nesses casos, prioriza-se o atendimento em UPHG, enfermarias psiquiátricas localizadas em estrutura de um hospital geral ${ }^{(4)}$. Os Hospitais Gerais são fundamentais na articulação entre os serviços da atenção à saúde mental de nível primário e serviços emergenciais e têm como função ofertar assistência em casos de emergências psiquiátricas, comumente caracterizadas por crises ou sintomas psíquicos agudizados ${ }^{(5-6)}$. Com isso, aumenta-se a oportunidade de integração entre a psiquiatria e a assistência à saúde em geral, evitando a estigmatização da doença mental e, consequentemente, a exclusão social do indivíduo ${ }^{(6)}$.

Embora as políticas de saúde mental preconizem o internamento de emergência psiquiátrica nestes serviços, elas ainda estão em processo de implementação, uma vez que há grande defasagem no número de UPHG em todo o Brasil. Em específico, no Paraná, dos 3.316 leitos psiquiátricos do estado, apenas 253 (7,62\%) são em Hospitais Gerais ${ }^{(7)}$.

Deste modo, as UPHG são consideradas serviços relativamente recentes na RAPS, estando, atualmente, em processo de expansão em todo o território brasileiro. Considerando este contexto, somada a escassez de pesquisas nestes serviços, ressalta-se a importância de conhecer o perfil sociodemográfico e clínico dos pacientes encaminhados às UPHG, a fim de possibilitar reflexões acerca do planejamento e da organização destes serviços de saúde e de políticas públicas, bem como ofertar aos profissionais de saúde subsídios para a implementação de uma assistência mais condizente com a realidade dessa clientela.

Com base no exposto, o objetivo deste estudo foi caracterizar o perfil sociodemográfico e clínico dos pacientes com transtorno mental, em tratamento em unidade de internação psiquiátrica de um hospital geral.

\section{MÉTODO}

Pesquisa descritiva transversal em duas unidades de internação psiquiátrica de um Hospital Geral (UPHG) do município de Guarapuava, Paraná, com 30 leitos para o sexo masculino e 30 para o feminino. Este município é o único da

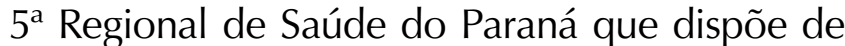
internação psiquiátrica, ofertando assistência a 20 municípios, além de atender outras demandas estaduais.

A amostra foi constituída por pacientes internados na UPHG, no período de $1^{\circ}$ de janeiro a 30 de junho de 2011. Entre os 461 pacientes internados neste período, 240 preencheram os seguintes critérios de inclusão: ser maior de 18 anos, ter comunicação verbal preservada, ter condições de entender a entrevista e responder às 
perguntas e expectativa de concluir o tratamento com alta médica programada.

Foram excluídos 221 pacientes: três menores de 18 anos, três indígenas, 13 não comunicativos, 31 sem condições de entender a entrevista e responder às perguntas, 49 por se recusarem a participar do estudo após serem abordados três vezes consecutivas e em momentos distintos, 66 não haviam concluído o tratamento e 56 tiveram alta não programada, o que inviabilizou a abordagem pela pesquisadora. A população indígena foi excluída por ser considerada área temática especial, conforme determina a Resolução CNS 196/96.

Os dados foram coletados na UPHG durante os meses de janeiro a junho de 2011, de segunda a sábado, no período diurno, conforme recomendação da coordenação da Unidade de Internação. Para tanto, foram feitas consultas aos prontuários e entrevista estruturada com os participantes do estudo durante o período de internação.

$\mathrm{O}$ instrumento estruturado apresentava questões para a coleta de informações referentes à caracterização sociodemográfica: idade, sexo, município de zona de residência, situação conjugal, raça/etnia, número de filhos, escolaridade, profissão/ocupação, renda; e clínica: idade de início do transtorno mental, primeiro diagnóstico psiquiátrico, diagnóstico psiquiátrico atual, tempo de diagnóstico, uso de substâncias psicoativas, comorbidade clínica e tratamento medicamentoso.

A pesquisa foi autorizada pela instituição e aprovada pelo Comitê de Ética em Pesquisa da Universidade Federal do Paraná, sob o ${ }^{\circ}$ 1016.141.10.09.

Os dados foram armazenados em um formulário do Google Docs ${ }^{\circledR}$ e enviados a uma planilha eletrônica de banco de dados. Os resultados foram analisados com o programa computacional Statistica ${ }^{\circledR}$ e expostos por frequências ou percentuais, médias, medianas, valores mínimos e máximos.

\section{RESULTADOS}

A idade dos 240 participantes - 84 mulheres e 156 homens - variou de 18 a 80 anos, com o grupo etário predominante entre 30 e 49 anos ( $n=137$ ), média de 41,5 anos, desvio padrão de $\pm 12,8$ e mediana de 41 . Declararam-se da raça branca $49,6 \%$ dos participantes $(n=119)$ e $72,1 \%$ ( $n=173)$ eram residentes da zona urbana. O estado civil prevalente dos entrevistados foi o de solteiro $(42,9 \%, n=103)$, e $66,3 \%(n=159)$ referiram ter pelo menos um filho (Tabela 1).

A escolaridade predominante dos participantes, Tabela 2, foi o ensino fundamental incompleto $(52,5 \%, n=126)$, com o percentual de abandono dos estudos de $77,1 \%$ ( $n=185)$, cujo principal motivo foi questões sociais. Apenas 41,7\% $(n=100)$ dos participantes se mantinham ativos no mercado de trabalho.

A Tabela 3 mostra que a idade de início do transtorno mental variou entre um e 78 anos, com média foi de 26,4 anos, desvio padrão de $\pm 13,1$ e mediana de 23. No que tange ao uso abusivo de substâncias lícitas, 79,5\% (n=224) dos indivíduos afimaram fazer uso. As comorbidades clínicas somaram 50,8\% ( $n=131)$, com pelo menos uma comorbidade. A ocorrência de comorbidades foi maior entre os homens entrevistados do que entre as mulheres, sendo a Hipertensão Arterial Sistêmica $(12,1 \%)$ a mais citada. 
Tabela 1 - Distribuição dos entrevistados segundo sexo e variáveis sociodemográficas. Guarapuava-PR, 2011

\begin{tabular}{|c|c|c|c|c|c|c|}
\hline \multirow{2}{*}{$\begin{array}{ll}\text { Variáveis } & \text { Sexo } \\
& \end{array}$} & \multicolumn{2}{|c|}{ Feminino } & \multicolumn{2}{|c|}{ Masculino } & \multicolumn{2}{|c|}{ Total } \\
\hline & $N(84)$ & $\%$ & $N(156)$ & $\%$ & $N(240)$ & $\%$ \\
\hline \multicolumn{7}{|l|}{ Idade } \\
\hline $18-29$ anos & 14 & 16,7 & 31 & 19,9 & 45 & 18,8 \\
\hline $30-39$ anos & 22 & 26,2 & 40 & 25,6 & 62 & 25,8 \\
\hline $40-49$ anos & 27 & 32,1 & 48 & 30,8 & 75 & 31,2 \\
\hline $50-59$ anos & 16 & 19 & 19 & 12,2 & 35 & 14,6 \\
\hline 60 anos ou mais & 5 & 6 & 18 & 11,5 & 23 & 9,6 \\
\hline \multicolumn{7}{|l|}{ Área de residência } \\
\hline Área Rural & 23 & 27,4 & 44 & 28,2 & 67 & 27,9 \\
\hline Área Urbana & 61 & 72,6 & 112 & 71,8 & 173 & 72,1 \\
\hline \multicolumn{7}{|l|}{ Situação Conjugal } \\
\hline Amasiado/Casado & 28 & 33,4 & 53 & 34 & 81 & 33,7 \\
\hline Separado & 16 & 19 & 25 & 16 & 41 & 17,1 \\
\hline Solteiro & 32 & 38,1 & 71 & 45,5 & 103 & 42,9 \\
\hline Viúvo & 8 & 9,5 & 7 & 4,5 & 15 & 6,3 \\
\hline \multicolumn{7}{|l|}{ Filhos } \\
\hline Possui & 63 & 75 & 96 & 61,5 & 159 & 66,3 \\
\hline Não Possui & 21 & 25 & 60 & 38,5 & 81 & 33,7 \\
\hline Total & 84 & 100 & 156 & 100 & 240 & 100 \\
\hline
\end{tabular}

Tabela 2 - Distribuição dos entrevistados segundo sexo, escolaridade, atividade ocupacional e renda. Guarapuava-PR, 2011

\begin{tabular}{|c|c|c|c|c|c|c|}
\hline \multirow{2}{*}{ Variáveis $\quad$ Sexo } & \multicolumn{2}{|c|}{ Feminino } & \multicolumn{2}{|c|}{ Masculino } & \multicolumn{2}{|c|}{ Total } \\
\hline & $N(84)$ & $\%$ & $N(156)$ & $\%$ & $N(240)$ & $\%$ \\
\hline \multicolumn{7}{|l|}{ Escolaridade } \\
\hline Analfabeto & 9 & 10,7 & 16 & 10,3 & 25 & 10,4 \\
\hline $\begin{array}{l}\text { Fundamental } \\
\text { Incompleto }\end{array}$ & 46 & 54,8 & 80 & 51,3 & 126 & 52,5 \\
\hline $\begin{array}{l}\text { Fundamental } \\
\text { Completo }\end{array}$ & 8 & 9,5 & 18 & 11,5 & 26 & 10,8 \\
\hline Médio Incompleto & 12 & 14,3 & 13 & 8,3 & 25 & 10,4 \\
\hline Médio Completo & 6 & 7,1 & 22 & 14,1 & 28 & 11,7 \\
\hline Superior Incompleto & 1 & 1,2 & 4 & 2,6 & 5 & 2,1 \\
\hline Superior Completo & 2 & 2,4 & 3 & 1,9 & 5 & 2,1 \\
\hline \multicolumn{7}{|l|}{ Atividade Profissional } \\
\hline Ativo & 53 & 63,1 & 47 & 30,1 & 100 & 41,7 \\
\hline Afastado/Inativo & 30 & 35,7 & 92 & 59 & 122 & 50,8 \\
\hline Aposentado & 1 & 1,2 & 17 & 10,9 & 18 & 7,5 \\
\hline \multicolumn{7}{|c|}{ Fonte de Renda Pessoal } \\
\hline Aposentadoria & 1 & 1,2 & 16 & 10,3 & 17 & 7,1 \\
\hline Benefício & 44 & 52,8 & 32 & 20,5 & 76 & 31,7 \\
\hline Emprego Fixo & 5 & 6 & 15 & 9,6 & 20 & 8,3 \\
\hline Não Possui & 26 & 30,4 & 42 & 26,9 & 68 & 28,3 \\
\hline Serviço Autônomo & 8 & 9,6 & 51 & 32,7 & 59 & 24,6 \\
\hline Total & 84 & 100 & 156 & 100 & 240 & 100 \\
\hline
\end{tabular}


Tabela 3 - Distribuição dos entrevistados de acordo com sexo, transtorno mental, medicação, comorbidades clinicas e substância psicoativa. Guarapuava-PR, 2011

\begin{tabular}{|c|c|c|c|c|c|c|}
\hline \multirow{2}{*}{$\begin{array}{ll} & \text { Sexo } \\
\text { Variáveis } & \\
\end{array}$} & \multicolumn{2}{|c|}{ Feminino } & \multicolumn{2}{|c|}{ Masculino } & \multicolumn{2}{|c|}{ Total } \\
\hline & $\mathbf{N}(84)$ & $\%$ & $\mathrm{~N}(156)$ & $\%$ & $\mathrm{~N}(\mathbf{2 4 0})$ & $\%$ \\
\hline \multicolumn{7}{|l|}{ Idade de início do Transtono mental } \\
\hline $0-10$ & 6 & 7,2 & 3 & 1,9 & 9 & 3,8 \\
\hline $10-19$ & 22 & 26,2 & 82 & 52,6 & 104 & 43,3 \\
\hline $20-29$ & 17 & 20,2 & 22 & 14,1 & 39 & 16,3 \\
\hline $30-39$ & 20 & 23,8 & 18 & 11,5 & 38 & 15,8 \\
\hline $40-49$ & 10 & 11,9 & 20 & 12,8 & 30 & 12,5 \\
\hline 50 ou mais & 6 & 7,1 & 9 & 5,8 & 15 & 6,3 \\
\hline Não sabe informar & 3 & 3,6 & 2 & 1,3 & 5 & 2,0 \\
\hline \multicolumn{7}{|l|}{ Primeiro Diagnóstico } \\
\hline $\mathrm{F} 10-\mathrm{F} 19$ & 14 & 16,7 & 92 & 59 & 106 & 44,2 \\
\hline $\mathrm{F} 20-\mathrm{F} 29$ & 16 & 19 & 30 & 19,2 & 46 & 19,2 \\
\hline F30 - F39 & 41 & 48,8 & 23 & 14,7 & 64 & 26,6 \\
\hline Outros & 13 & 15,5 & 11 & 7,1 & 24 & 10 \\
\hline \multicolumn{7}{|l|}{ Diagnóstico Atual* } \\
\hline F10 - F19 & 15 & 17,9 & 99 & 63,5 & 114 & 47,5 \\
\hline F20 - F29 & 50 & 59,4 & 49 & 31,4 & 99 & 41,3 \\
\hline $\mathrm{F} 30-\mathrm{F} 39$ & 15 & 17,9 & 5 & 3,2 & 20 & 8,3 \\
\hline Outros & 2 & 2,4 & 2 & 1,3 & 4 & 1,6 \\
\hline Sem diagnóstico & 2 & 2,4 & 1 & 0,6 & 3 & 1,3 \\
\hline Uso de Substâncias Psicoativas** & $\mathrm{N}(52)$ & $\%$ & $N(229)$ & $\%$ & $N(281)$ & $\%$ \\
\hline Álcool e Tabaco & 43 & 82,7 & 181 & 79 & 224 & 79,7 \\
\hline Maconha & 4 & 7,8 & 10 & 4,4 & 14 & 5 \\
\hline Maconha e Crack & 1 & 1,9 & 7 & 3,2 & 8 & 2,8 \\
\hline Maconha Crack e Cocaína & 1 & 1,9 & 6 & 2,6 & 7 & 2,5 \\
\hline Crack & 1 & 1,9 & 6 & 2,6 & 7 & 2,5 \\
\hline Cocaína, Solvente, Crack e Maconha & 1 & 1,9 & 5 & 2,2 & 6 & 2,1 \\
\hline $\begin{array}{l}\text { Ecstasy, LSD, Cocaína, Solvente, } \\
\text { Crack e Maconha }\end{array}$ & - & - & 5 & 2,2 & 5 & 1,8 \\
\hline Maconha e Cocaína & - & - & 4 & 1,7 & 4 & 1,4 \\
\hline Cocaína & - & - & 4 & 1,7 & 4 & 1,4 \\
\hline Solvente, Crack e Maconha & 1 & 1,9 & 1 & 0,4 & 2 & 0,8 \\
\hline Tempo de Diagnóstico & $\mathrm{N}(84)$ & $\%$ & $N(156)$ & $\%$ & $\mathrm{~N}(240)$ & $\%$ \\
\hline $1^{\circ}$ diagnóstico & 12 & 14,3 & 20 & 12,8 & 32 & 13,3 \\
\hline $1-5$ anos & 14 & 16,7 & 28 & 17,9 & 42 & 17,5 \\
\hline $6-10$ anos & 12 & 14,3 & 24 & 15,4 & 36 & 15 \\
\hline $11-20$ anos & 14 & 16,7 & 33 & 21,2 & 47 & 19,6 \\
\hline 20 anos ou mais & 29 & 34,5 & 49 & 31,4 & 78 & 32,5 \\
\hline Não informado & 3 & 3,6 & 2 & 1,3 & 5 & 2,1 \\
\hline \multicolumn{7}{|l|}{ Tratamento medicamentoso } \\
\hline Sim & 53 & 63,1 & 78 & 50 & 131 & 55,6 \\
\hline Não & 31 & 36,9 & 78 & 50 & 109 & 45,4 \\
\hline \multicolumn{7}{|l|}{ Comorbidade Clínicas } \\
\hline Sim & 51 & 60,7 & 71 & 45,5 & 122 & 50,8 \\
\hline Não & 33 & 39,3 & 85 & 54,5 & 118 & 49,2 \\
\hline
\end{tabular}

*F10 - F19: transtornos mentais e comportamentais devidos ao uso de substância psicoativa; F20-F29: Esquizofrenia, transtornos esquizotípicos e transtornos delirantes; F30-F39: Transtornos do humor (afetivos); **Foi considerado o uso de mais de uma substância psicoativa por participante. 


\section{DISCUSSÃO}

De modo geral, o perfil dos pacientes entrevistados nesta pesquisa se assemelha ao perfil encontrado em outro estudo também feito em um serviço de emergências psiquiátricas de um hospital geral universitário, localizando na cidade de Sobral, Ceará, com informações dos prontuários de 191 pacientes $^{(5)}$.

No que se refere à faixa etária, a maioria correpondeu a adultos, fase mais longa da vida, em que deve acontecer o auge das realizações pessoais como casamento, criação dos filhos e atividades laborais, intensamente valorizadas nesta idade; no entanto, a presença de trantornos mentais nessa faixa etária influencia, muitas vezes, de forma negativa, o modo de vida, pois interrompe a produtividade da pessoa pela sua cronicidade $^{(5,8)}$.

Um estudo ${ }^{(9)}$ sobre o perfil sociodemográfico e clínico de pacientes em internações psiquiátricas na cidade de Fortaleza, Ceará, mostrou que a média de idade de procura por tratamento foi de 37,3 anos, similar a este estudo, fato que deve ser visto como preocupante, considerando seus desdobramentos.

Quanto à distribuição das pessoas de acordo com o local de residência, a maioria residia na área urbana. De acordo com dados do Instituto Brasileiro o e Geografia e Estatística (IBGE), a população do Estado do Paraná é composta por, aproximadamente, $85 \%$ de pessoas residentes na área urbana. De modo geral, os estudos que tratam dos transtornos mentais não mostram correlação entre a prevalência desses transtornos e a área de residência ${ }^{(10)}$.

O contingente dos participantes que vivem sem um companheiro foi predominante, com a ênfase no estado civil solteiro. Este dado se confirma em pesquisa com pacientes em tratamento em UPHG, cuja prevalência chegou a $74,8 \%{ }^{(5)}$.

Outro dado relevante que emergiu nessa pesquisa é que a maioria tinha filhos, realidade que pode ser comparada a outra pesquisa com pacientes portadores de transtorno mental ${ }^{(11)}$. No entanto, a literatura pesquisada não evidencia correlações entre ter ou não filhos e a ocorrência de transtornos mentais. É importante destacar que os trantornos mentais afetam não somente o indivíduo, mas todos os seus familiares, em particular, os filhos ${ }^{(12)}$.

Por sua vez, nos transtornos mentais relacionados a substâncias psicoativas, o vínculo afetivo entre cônjuges e filhos é afetado consideravelmente. Do mesmo modo, as relações interpessoais são agravadas, principalmente pelas mudanças de comportamento causadas pelo abuso das substâncias psicoativas, bem como os problemas sociais e legais que este abuso pode gerar. Em tais situações podem surgir nos conviventes do dependente químico a desesperança, a perda do contato físico e os sintomas que reportam a transtornos do humor, sobretudo à depressão(13).

A escolaridade predominante dos participantes foi o ensino fundamental incompleto, que corresponde a um período inferior a nove anos de estudo, de acordo com o Sistema Educacional Brasileiro ${ }^{(10)}$. A predominância da baixa escolaridade da população estudada é explicada pelo fato de a maioria dos entrevistados ter abandonado os estudos.

Esses dados se assemelham aos resultados de estudos feitos em diversos serviços de saúde mental ${ }^{(14-15)}$. Estudo feito no Piauí, em um CAPS ad, com o prontuário de 1043 pacientes, mostrou que $40,1 \%$ tinham o ensino fundamental ${ }^{(15)}$. Em estudo feito em Ribeirão Preto, São Paulo, com 54 pessoas com trantornos mentais, constatou-se que $50 \%$ haviam estudado somente até o ensino fundamental, enquanto $39,6 \%$, até o ensino médio, sendo que somente $20,8 \%$ havia conluído seus estudos ${ }^{(14)}$.

Quanto à atividade laboral, a maioria das pessoas estudadas referiu estar ativo no mercado de trabalho, porém houve um quantitativo relevante de abandono do emprego. Esta característica pode estar relacionada ao fato de que, ao considerar o total de pessoas pesquisadas com diagnóstico de transtornos mentais, principalmente aquelas relacionadas ao uso de drogas, os participantes, possivelmente, apresentam diversos prejuízos sociais relacionados ao mercado de trabalho, o que pode ter contribuído para seu abandono.

Contudo, compreende-se, pelos dados pesquisados, que a principal fonte de renda dos pacientes é o Benefício Previdenciário, similar ao de outros estudos ${ }^{(16-17)}$ com pessoas portadoras de transtorno mental no que concerne ao emprego formal e recebimento de aposentadoria. 
Nesse sentido, cabe a discussão sobre os custos previdenciários dos pacientes com transtorno mental, bem como as possibilidades de inclusão dessas pessoas no mercado de trabalho. Na IV Conferência Nacional de Saúde Mental foi discutida a implantação de uma política de inclusão produtiva no mercado formal de trabalho, embasada na integralidade e na intersetorialidade, o que significa envolver Ministérios da Saúde e da Previdência Social em ações conjuntas que visem à diminuição dos custos previdenciários e garantam a inclusão ${ }^{(18)}$.

O perfil clínico desses pacientes destaca que o primeiro diagnóstico de transtorno mental ocorreu na adolescência, período caracterizado por mudanças corporais, principalmente relacionadas ao desenvolvimento sexual, consolidação da personalidade e transformações sociais, que compreendem um momento de preparação interna para a vida adulta. Nesta fase, pode ocorrer uma crise de identidade, considerada normal, mas que pode interferir no aparecimento de transtorno mental, caso não seja adequadamente administrada $^{(8)}$.

Os transtornos mentais e comportamentais devidos ao uso substâncias psicoativas são apontados no cenário epidemiológico nacional de maneira preocupante, pois a experimentação dessas substâncias está cada vez mais precoce, tornando seu uso ainda mais prejudicial nos aspectos biológico e social, por sua relação com comportamento de risco individual e coletivo ${ }^{(19)}$.

No período pesquisado, para o sexo masculino, os principais diagnósticos de internamento foram os de transtornos mentais e comportamentais devidos ao uso de substâncias psicoativas. Embora a literatura aponte aumento no consumo, de maneira geral, o maior uso e suas consequências mais significativas são ainda comuns na população masculina. Isso ocorre porque, tradicionalmente, os homens se sentem imunes às diversas enfermidades biológicas e mentais, por conseguinte diminuindo a adesão a medidas preventivas e a procura por serviços primários por essa parcela da população ${ }^{(20)}$.

$\mathrm{Na}$ UPHG em estudo, as pessoas com transtorno mental causado pelo uso de substância psicoativa são tratadas no mesmo ambiente das pessoas com transtornos psicóticos, de humor e outros. Evidenciou-se que, além das pessoas que estão em tratamento para dependência, consomem algum tipo de substância psicoativa lícita.

De acordo com o II Levantamento domiciliar sobre o uso de drogas psicotrópicas no Brasil, em relação ao sexo feminino, as pessoas do sexo masculino fazem mais uso de álcool durante a vida, substância que também se destacou no presente estudo. Quanto ao tabaco, o mesmo levantamento traz um quantitativo ligeiramente maior de homens em relação às mulheres ${ }^{(2)}$.

A disponibilidade de álcool e drogas cada vez maior é fator que pode estar associado ao aumento do uso dessas substâncias pelos portadores de transtornos mentais graves. Há, neste caso, necessidade de identificar se as pessoas portadoras de outros transtornos não relacionados ao álcool abusam de bebidas alcoólicas ou são delas dependentes, identificando, assim, a comorbidade ${ }^{(21)}$.

Quanto às mulheres, o diagnóstico de maior prevalência foi esquizofrenia, transtornos esquizotípicos e delirantes (F20 - F29), fato que difere da literatura pesquisada, a qual aponta, no sexo feminino, maior prevalência do grupo de transtornos do humor (F30 -F39), como depressão e transtorno bipolar ${ }^{(12)}$.

Em relação ao tempo de diagnóstico, houve predominância de 20 anos ou mais. Neste contexto, a literatura ressalta que os transtornos mentais são os que mais trazem incapacidades e anos de vida perdidos por se tratar de doença crônica ${ }^{(22)}$.

A ocorrência de tratamento medicamentoso foi frequente, tendo a maioria dos participantes referida à utilização de algum medicamento psicotrópico ao longo da vida. Entre as mulheres, o uso de medicamento é mais frequente em relação aos homens, $63,1 \%$ e $50 \%$, respectivamente.

A grande utilização dos psicofármacos pode ser justificada pelo seu uso no controle e diminuição dos sintomas dos transtornos mentais, sobretudo naqueles que se manifestam pela psicose e pelo humor deprimido. Porém, é necessário que o tratamento medicamentoso seja combinado com tratamentos não medicamentosos, entre eles, a psicoterapia $^{(11)}$.

O fato de $45,4 \%$ dos participantes responderem que ao longo da vida não utilizaram nenhum psicofármaco pode estar relacionado ao alto número de internações de pessoas dependentes de substâncias psicoativas. Estas pessoas se beneficiam do medicamento somente nos quadros 
de intoxicação e de sintomas de abstinência, mas geralmente isso ocorre em âmbito hospitalar ${ }^{(23)}$.

Sobre as comorbidades clínicas, o fato de os homens entrevistados apresentarem um percentual maior de comorbidade do que as mulheres pode também estar relacionado ao diagnóstico psiquiátrico, pois o uso de substâncias psicoativas pode contribuir para o aparecimento de doenças cardiovasculares, pulmonares e hepáticas ${ }^{(19)}$.

Há uma associação importante entre transtornos mentais e comorbidades clínicas, pessoas com problemas físicos, como os problemas cardiovasculares e endócrinos, podem desenvolver transtornos depressivos, ansiosos, entre outros, evidenciando a importância da integralidade do cuidado prestado a essas pessoas $^{(12)}$.

O pensamento dualista disseminado na medicina incide diretamente na relação dos transtornos mentais e doenças clínicas, constituindo, para os profissionais de saúde, uma prática difícil o reconhecimento de doenças clínicas em pessoas com transtorno mental e vice-versa. Neste sentido, é necessário que estes profissionais reconheçam a existência de possíveis comorbidades para proporcionar tratamento adequado para ambas as condições vividas pela pessoa com transtorno mental.

\section{CONSIDERAÇÕES FINAIS}

Os resultados obtidos permitiram delinear um perfil de pessoas do sexo masculino em idade produtiva, brancos, residentes na área urbana, solteiros, com filiação e ativos no mercado de trabalho. A escolaridade correspondeu ao ensino fundamental incompleto, com histórico de abandono dos estudos.

Foram observadas ligações entre a situação socioeconômica dos participantes e questões relacionadas aos transtornos mentais, com prejuízo de forma individual e coletiva. A baixa escolaridade foi relacionada à incapacidade provocada por transtornos mentais e ao uso de substâncias psicoativas.

Quanto ao perfil clínico, foi possível considerar que os transtornos mentais se manifestaram na adolescência, estando relacionado ao uso de drogas, principalmente no sexo masculino, fato que se refletiu no diagnóstico atual de trantorno mental. O uso de medicação esteve presente em uma parcela significativa dos pacientes estudados neste trabalho, bem como o desenvolvimento de comorbidades clínicas.

Com relação ao trabalho do enfermeiro e da equipe multiprofissional de saúde mental, entende-se que conhecer esse perfil auxilia no planejamento das ações de saúde mental, tanto na instituição de internação, como na comunidade, por meio da atenção primária. Além disso, pode permitir e fortalecer as relações intersetoriais, haja vista que o cuidado em saúde mental deve ser de corresponsabilidade dos setores de saúde, assistência social, justiça e educação.

\section{REFERÊNCIAS}

1. World Health Organization. Investing in mental health: evidence for action. Geneva: World Health Organization; 2013.

2. Ministério da Saúde (BR). Direitos humanos e saúde mental. [Internet] 27 nov 2014 [acesso em 27 nov 2014]. Disponível em: http://www.sdh.gov.br/assuntos/ direito-para-todos/programas/direitos-humanos-emsaude-mental

3. Ministério da Saúde (BR). Portaria n. 3.088, de 23 de dezembro de 2011. Institui a rede de atenção psicossocial para pessoas com sofrimento ou transtorno mental e com necessidades decorrentes do uso do crack, álcool e outras drogas, no âmbito Sistema Único de Saúde. Brasília: Ministério da saúde; 2011.

4. Silva NG, Silva PP, Oliveira AGB. A percepção dos trabalhadores de enfermagem sobre a assistência à saúde mental em hospital universitário. Cienc. cuid. saúde. 2012;11(2): 302-310.

5. Sousa FSS, Silva CAF, Oliveira EN. Serviço de emergência psiquiátrica em hospital geral: estudo retrospectivo. Rev. Esc. Enferm. USP. 2010;44(3):796802.

6. Paes MR, Silva TL, Chaves MMN, Maftum MA. O papel do hospital geral na rede de atenção à saúde mental no Brasil. Cienc. cuid. saúde. 2013; 12(2): 407-412.

7. Brasil. Ministério da Saúde. Departamento de Informática do Sistema Único de Saúde (DATASUS). Ministério da Saúde, [Internet] 17 mai 2010 [acesso em 17 mai 2010]. Disponível em:http:// www.datasus. gov.br

8. Oliveira RPO, Laus AM. Caracterização de pacientes 
de unidade de internação psiquiátrica, segundo o grau de dependência do cuidado de enfermagem. Rev Esc Enferm USP. 2011; 45(5):1164-70.

9. Oliveira MSN, Pinto FJM, Aguiar JB, Sampaio RMM, Medeiros CRB. Perfil sociodemográfico e clínico de pacientes em internações psiquiátricas voluntárias e involuntárias. Rev Bras Promoç Saúde. 2011;24(4):361-66.

10. Instituto Brasileiro de Geografia e Estatística [Internet]. Brasília: Ministério do Planejamento, Orçamento e Gestão (BR) [cited 2012 jul 27]. Censo demográfico 2010: Características da População e dos Domicílios. Available from: http://www.ibge.gov.br/home/ estatistica/populacao/censo2010/caracteristicas_da_ populacao/resultados_do_universo.pdf

11. Cardoso L, Galera SAF. Doentes Mentais e seu perfil de adesão ao tratamento psicofarmacológico. Rev. esc. enferm, USP. 2009;43(1):161-167.

12. Botti NCL, Ferreira SC, Nascimento RG, Pinto JAF. Condições de saúde de mulheres com trantornos mentais. 2013;14(6):1209-16.

13. Aragão ATM, Milagres E, Figlie NB. Qualidade de vida e desesperança em familiares de dependentes químicos. Psico-USF. 2009;14(1):117-123.

14. Cardoso L, Galera SAF. Internação psiquiátrica e manutenção do tratamento extra-hospitalar. Rev Esc Enferm USP. 2011;45(1):87-94.

15. Monteiro CFS, Fé LCM, Moreira MAC, Albuquerque IEM, Silva MG, Passamani MC. Perfil sociodemográfico e adesão ao tratamento de dependentes de álcool em CAPS-AD do Piauí. Esc Anna Nery. 2011;15(1):90-95.

16. Machado V, Santos MA. Taxa de permanência hospitalar de pacientes reinternados em hospital psiquiátrico. J. Bras Psiquiatr. 2011;60(1):16-22.

17. Siano AK, Ribeiro LC, Ribeiro MS. Análise comparativa do registro médico-pericial do diagnóstico de transtornos mentais de segurados do Instituto Nacional do Seguro Social requerentes de auxílio-doença. J Bras Psiquiatr. 2010;59(2):131-138.

18. Ministério da Saúde. Sistema Unico de Saúde. Conselho Nacional de Saúde. Comissão Organizadora da IVConferência Nacional de Saúde Mental- Intersetorial. Relatório Final da IV Conferência Nacional de Saúde Mental. Brasília (BRASIL): Conselho Nacional de Saúde/Ministério da Saúde, 2010.

19. Azevedo RCS, Silva VF. Dependência de substâncias psicoativas: conceitos básicos. In: Bodega NJ. Prática psiquiátrica no hospital geral: interconsulta e emergência. Porto Alegre: Artmed, 2012. 356-371.

20. Ministério da Saúde. Política Nacional de Atenção à Saúde do Homem: princípios e diretrizes.
Brasília(BRASIL): 2009. Available from: http://portal. saude.gov.br/portal/arquivos/pdf/politica_nacional_ atencao_integral.pdf

21. Hess ARB, Almeida RMM, Moraes AL. Comorbidades psiquiátricas em dependentes químicos em abstinência em ambiente protegido. Estudos de Psicologia. 2012;17(1): 171-8.

22. Grigolatto T, Chagas MHN, Trzesniak C, Crippa JAS, Hallak JEC, Santos JLF. A influência dos papéis sociais na qualidade de vida de portadores de esquizofrenia. Cad. Ter.Ocup. UFSCar. 2014;22(1): 195-203.

23. Owen JÁ, Ferrando S, Levenson JL. Psicofármacos: interações medicamentosas. In: Botega, NJ. Prática psiquiátrica no hospital geral: interconsulta e emergência. Porto Alegre: Artmed, 2012. 556-584. 